\title{
Mathematical Model for Image Restoration Based on Fractional Order Total Variation
}

\author{
Akhtar Rasool ${ }^{1}$, Noor Badshah², Asmat Ullah ${ }^{3}$ \\ ${ }^{2,}{ }^{1}$ Basic Sciences Department, University of Engineering and Technology KP Peshawar (Pak). \\ ${ }^{3}$ Department of Engineering Mechanics, Hohai University, No.1 Xikang Road, Nanjing, Jiangsu 210098, PR China.
}

\begin{abstract}
This paper addresses mathematical model for signal restoration based on fractional order total variation (FOTV) for multiplicative noise. In alternating minimization algorithm the Newton method is coupled with time-marching scheme for the solutions of the corresponding PDEs related to the minimization of the denoising model. Results obtained from experiments show that our model can not only reduce the staircase effect of the restored images but also better improve the PSNR as compare to other existed methods.
\end{abstract}

Keywords- Total Variation (TV), Fractional Order Derivative, Speckle Noise, Image Restoration.

\section{INTRODUCTION}

Image denoising has various applications in different fields including pattern recognition, remote sensing, preprocessing for computer vision and medical images. It is still a valid challenge for researchers. Two important types of image denoising are additive noise and multiplicative noise $[1,2]$. In additive noise removal model, the original image $u$ is considered to be contaminated by Gaussian additive noise $n$. Our goal is to restore $u$ from $\mathrm{g}=\mathrm{u}+\mathrm{n}$. The total variation (TV) has been developed by Rudin, Osher and Fatemi. TV based method is edge preserving [1]. We discuss multiplicative noise in this paper. Consider the noisy image $g: \Omega \rightarrow \mathrm{R}$, the original image $u$ is contaminated by some noise $v$ satisfying the Gamma law with mean 1 . This type of noise mostly exists in ultrasound imaging, sonar and laser imaging and synthetic aperture radar images. Multiplicative noise are more complex as compared to additive noise. Rudin et al early approached a variational model [3]. Second approach for multiplicative noise has been proposed by Aubert and Aujol, see [4] for more details. Keeping in mind the statistical properties of multiplicative noise $n$, we approximate $g$ by treating the following constrained minimization problem

$$
\min _{u} \int_{\Omega}|D u| d x d y
$$

Subject to: $\int_{\Omega} \frac{g}{u} d x d y=1, \int_{\Omega}\left(\frac{g}{u}-1\right)^{2} d x d y=\sigma^{2}$.
The mean of noise is 1 with standard deviation $\sigma^{2}$ as listed above. TV regularization methods remove noise and simultaneously preserve sharp intensity boundaries but sometimes textures are smoothed with noise in noise removing process [1-3]. The staircase effects can be reduced by generalizing the regularization term which can be performed in two ways. First approach is based on high order derivative; see [5-7].

Secondly, the regularization term can be generalized by using fractional-order derivatives. In recent past, some general fractional-order additive noise models have been treated in $[8,9]$. Chambolle used the fitting term $\|g-u\|_{2}^{2}$ in ROF model and hence introduced an efficient projection method for additive noise [10]. Obtained results indicate that the performance of fractional-order TV are better than integer high order TV. Fractional order models have competitive performance with other integer order based models in noise removing as well as staircase reduction. Moreover Aubert and Aujol developed a multiplicative noise model "AA-model", which is given by

$$
\min _{u} \int_{\Omega}\left(\log u+\frac{g}{u}\right) d x d y+\lambda \int_{\Omega}|D u| d x d y
$$

Recently Zhang Jun and Wei Zhihui introduced fractional order AA Model by replacing TV term in AA model with fractional order TV.

As the function $\left(\log u+\frac{\mathrm{g}}{\mathrm{u}}\right)$ is not convex everywhere, therefore Haung et al [11] introduced the auxiliary variable $\mathrm{w}=\operatorname{logu}$ and introduced a strictly convex function

$$
\begin{aligned}
& \min _{w, u} \int_{\Omega}\left(w+g e^{-w}\right) d x d y+\alpha_{1}|w-u|_{L_{2}}^{2}+ \\
& \alpha_{2} \int_{\Omega}|D u| d x d y,
\end{aligned}
$$

with two regularization parameters $\alpha_{1}$ and $\alpha_{2}$. As $u=e^{-w}$ is strictly convex for all value of $w$ which ensures that the solution of the variational problem is unique. TV based models have better performance in preserve sharp edges, reducing the noise but often these models cause the staircase effect. We develop fractional-order TV based method for multiplicative noise in Sect. 2. In Sect. 3, we use alternating minimization algorithm to find minimizer of our proposed problem .In Sect. 4, we give some numerical results to validate 
the performance of the proposed methodology. Finally, the paper is concluded in the last Section.

\section{THE PROPOSED APPROACH}

We introduce a convex function for restoring images contaminated by multiplicative noise in this section. Our new model is based on fractional Order AA model and HNW algorithm. Replacing TV term in model (3) with Fractional order TV, we introduce the following model.

$$
\begin{array}{r}
\min _{w, u} \int_{\Omega}\left(w+g e^{-w}\right) d x d y \\
+\alpha_{1}|| w-\left.u\right|_{L_{2}} ^{2}+\left(\alpha_{2}+\alpha_{3} u^{2}\right)\left|D^{\alpha} u\right|,
\end{array}
$$

with three positive regularization parameters $\alpha_{1}, \alpha_{2}$ and $\alpha_{3}$. The fractional-order total variation norm provides a better performance in preserving image edges. It also reduces staircase effect. Moreover $u$ and $w$ preserve edges at the same point; see [9]. The use of fractional-order TV to $w$ is quite better for preserving sharp edges and reducing staircase effect. The main advantage is that $\mathrm{u}=\mathrm{e}^{\mathrm{w}}$ is positive for all $w$, even though $u$ in the objective function (2) required to be positive. In our proposed model if we assign $w$ a large negative number, $u$ is still a small non zero positive number.

\section{NUMERICAL SCHEME}

We use an alternating minimization algorithm to solve the FOTV problem. We split eqn. (4) in the following two subproblems.

(i) First fix $u$ and find the solution of

$\min _{\mathrm{w}} \int_{\Omega}\left(\mathrm{w}+\mathrm{ge}^{-\mathrm{w}}\right) \mathrm{dxdy}+\alpha_{1}\|w-u\|_{\mathrm{L}_{2}}^{2}$.

(ii) Then fix $w$, find the solution of

$$
\begin{aligned}
& \min _{\mathrm{u}} \alpha_{1}|| \mathrm{w}-\left.\mathrm{u}\right|_{\mathrm{L}_{2}} ^{2} \\
& +\left(\alpha_{2}+\alpha_{3} \mathrm{u}^{2}\right) \int_{\Omega}\left|\mathrm{D}^{\alpha} \mathrm{u}\right| \mathrm{dxdy} .
\end{aligned}
$$

Applying discrete setting, the first problem gets the form:

$\min _{\mathrm{w}} \sum_{1}^{n^{2}}\left(w_{i}+g e^{-w_{i}}\right)+\alpha_{1}|| w-u \|_{2}^{2}$.

The minimizer of the above problem can be obtained by solving the following nonlinear system

$1-g e^{-w_{i}}+2 \alpha_{1}\left(w_{i}-u_{i}\right)=0, i=1,2, \ldots n^{2}$.

The 2nd derivative w.r.t $w$ of the first term in (5) is $g e^{-w}$, which is positive for positive value of $g$. Thus the strictly convex function (5) has a unique minimum which we compute with Newton method as

$$
\mathrm{w}_{\mathrm{i}+1}=\mathrm{w}_{\mathrm{i}}-\frac{1-\mathrm{ge}^{-\mathrm{w}_{\mathrm{i}}}+2 \alpha_{1}\left(\mathrm{w}_{\mathrm{i}}-\mathrm{u}\right)}{\mathrm{ge}^{-\mathrm{w}_{\mathrm{i}}}+2 \alpha_{1}}, \quad \mathrm{i}=0,1,2, \ldots
$$

The Euler-Lagrange equation of FOTV model (6) is

$$
\begin{array}{r}
\frac{\partial u}{\partial t}=\frac{2 \alpha_{1}}{\alpha_{2}+\alpha_{3} u^{2}}\left(w_{i, j}^{n}-u_{i, j}\right)+(-1)^{\alpha} \operatorname{div}^{\alpha} \frac{\nabla^{\alpha} u_{i, j}}{\left|\nabla^{\alpha} u_{i, j}\right|} \\
\frac{\partial u}{\partial n}=0 \text { on } \partial \Omega, \quad \mathrm{u}(0)=\mathrm{u}_{0} .
\end{array}
$$

The numerical scheme for the steady-state equation (9) is given by

$$
u_{i, j}^{n+1}=u_{i, j}^{n}+\eta \tau\left|w_{i, j}-u_{i, j}\right|+(-1)^{\alpha} d i v^{\alpha} \frac{\nabla^{\alpha} u_{i, j}}{\left|\nabla^{\alpha} u_{i, j}\right|},
$$

$\eta=\frac{2 \alpha_{1}}{\alpha_{2}+\alpha_{3} u^{2}}$ with B. Conditions

$$
u_{0, j}^{n}=u_{1, j}^{n}, \quad u_{N, j}^{n}=u_{N-1, j}^{n},
$$

$u_{i, 0}^{n}=u_{i, 1}^{n}, u_{i, N}^{n}=u_{i, N-1}^{n}$.

Algorithm

We take initial guess $u^{(0)}$ to be the noisy image.

(i) First fix $u^{(k-1)}$, compute by Newton's algorithm $w^{(k)}=$ $\arg \min _{\mathrm{w}} \sum_{k=0}^{n^{2}}\left(w_{i}+g e^{-w_{i}}\right)+\alpha_{1}\left\|w-u^{k-1}\right\|_{2}^{2}$

(ii) Then fix $w^{(k)}$, compute $u^{(k)}$ by using

$$
\frac{\partial u}{\partial t}=\frac{2 \alpha_{1}}{\alpha_{2}+\alpha_{3} u^{2}}|| w^{(k)}-u||+(-1)^{\alpha} \operatorname{div}^{\alpha}\left(\frac{\nabla^{\alpha} u}{\left|\nabla^{\alpha} u\right|}\right)
$$

(iii) Exit with an approximate denoised image $u^{k}=e^{w^{(k)}}$ if the stopping criteria $\frac{|| u^{k+1}-u^{k}||}{\|\left. u^{k+1}\right|_{2}}<10^{-4}$ is satisfied, otherwise we continue with $k=k+1$ for next iteration.

\section{NUMERICAL RESULTS}

This section describes some experimental results of image denoising to claim the superiority of our method. We consider some images contaminated by multiplicative noise. Figure 1 shows clean images. Results obtained from experiments indicate the superiority of our proposed method. We use PSNR to compare the capabilities of the restored images as

$$
P S N R=20 \log _{10} \frac{255}{\left\|u-u_{\text {true }}\right\|}
$$

Where $u_{\text {true }}$ and $u$ denote the noise-free signal and recovered signal respectively. 
Original Images

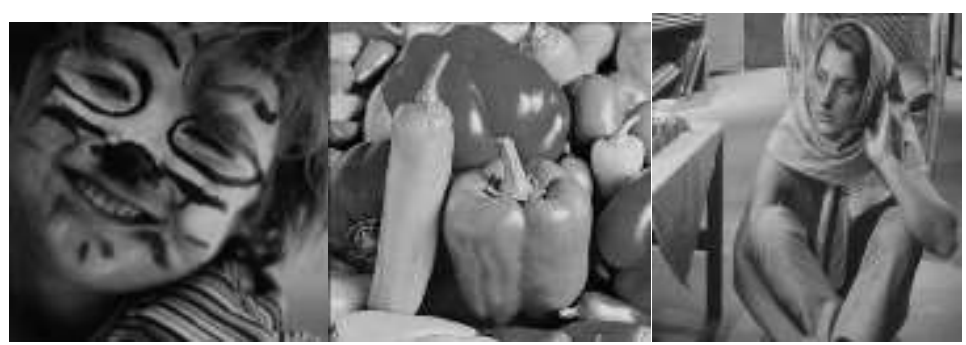

Fig. 1: Face, Peppers, Barbara

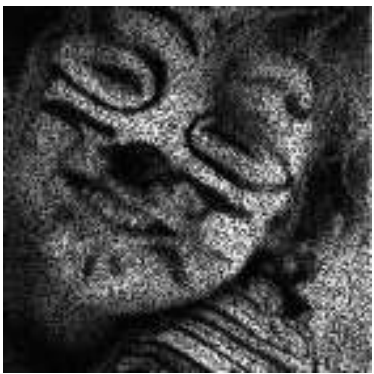

(a) Face 0.3

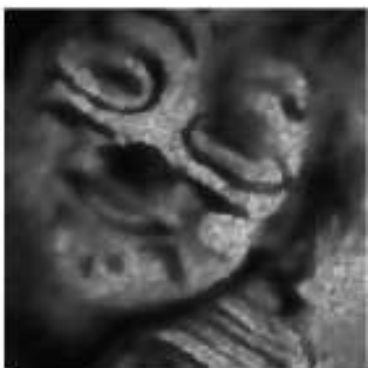

(b) AA Method

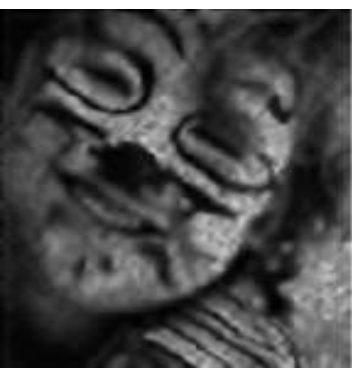

(c) Proposed Method

Fig.2: (a). Noisy Image, $\sigma^{2}=0.3$ (b) Restored signal "AA-method", $\lambda=35$

(c) Restored signal "Proposed method", $\alpha_{1}=$ $190, \alpha_{2}=0.00002 a_{3}=490$.

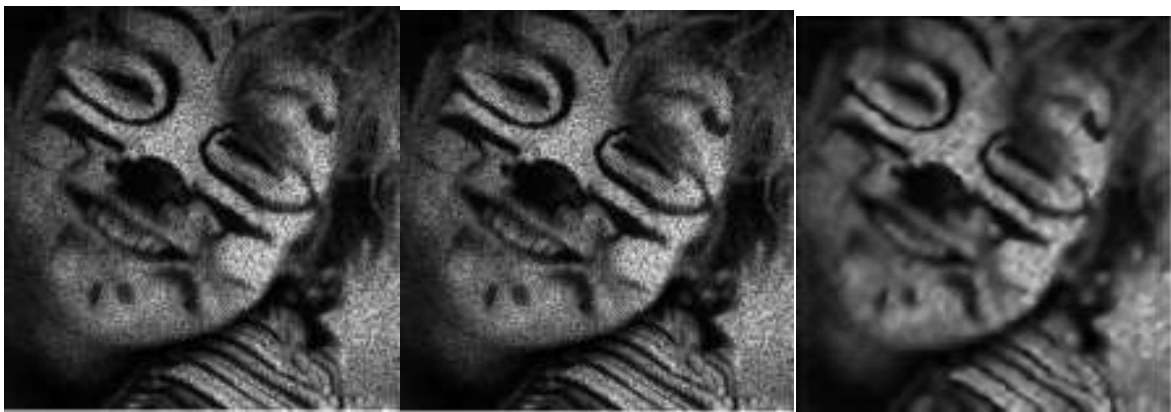

(a) Face, $\sigma=0.1(b) A A-M e t h o d$ (c) Proposed Method

Fig 3 (a) Noisy Signal, $\sigma^{2}=0.1\left(\right.$ b) Restored Signal, AA-Method, $\lambda=35$ (c) Restored signal "Proposed method", $\alpha_{1}=$ $190, \alpha_{2}=0.00002 a_{3}=490$.

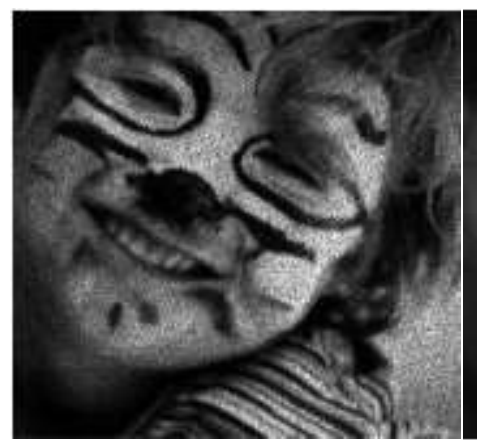

(a) Noisy Image, $\sigma=0.03$

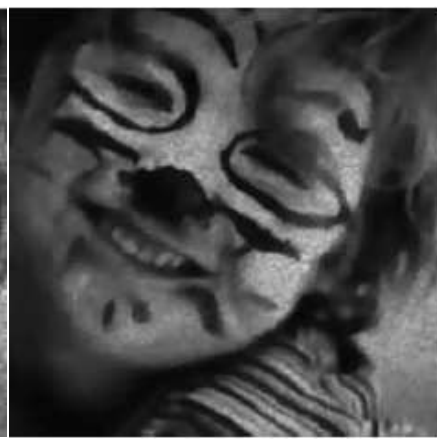

(b) AA Method

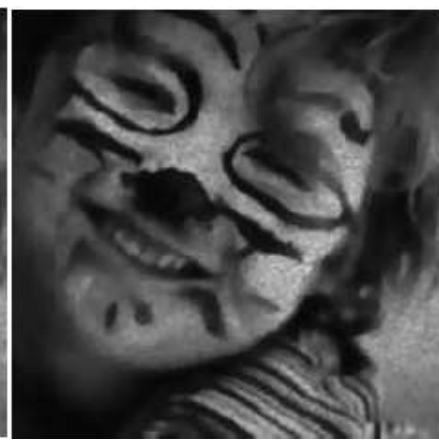

(c) Proposed Method

Fig.4:(a) Noisy Signal, $\sigma^{2}=0.03$. (b) Restored Signal, AA-Method, $\lambda=35$ (c) Proposed Method, $\alpha_{1}=16, \alpha_{2}=0.0002, \alpha_{3}=$ 150. 


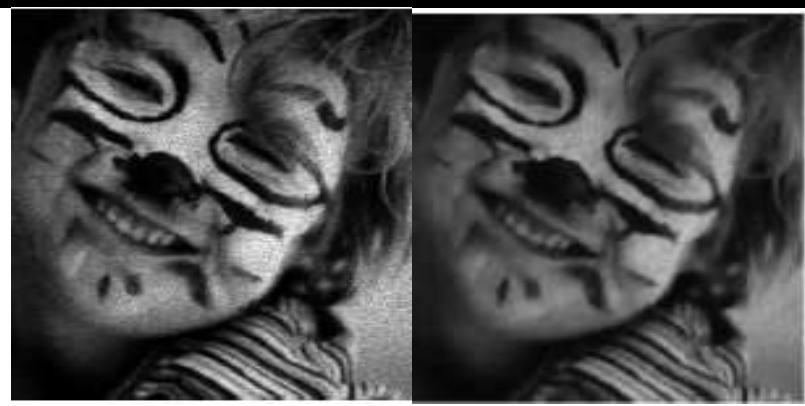

Test 1: (a) Noisy Image, $\sigma=0.03 \quad$ (b) AA Method

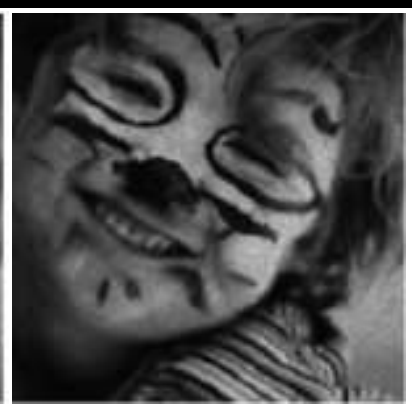

(c) Proposed Method

Fig.5: (a) Noisy Signal, $\sigma^{2}=0.01$. (b) Restored Signal, AA-Method, $\lambda=34$ (c) Proposed Method, $\alpha_{1}=12, \alpha_{2}=0.0002, \alpha_{3}=$ 490.

Test 2.

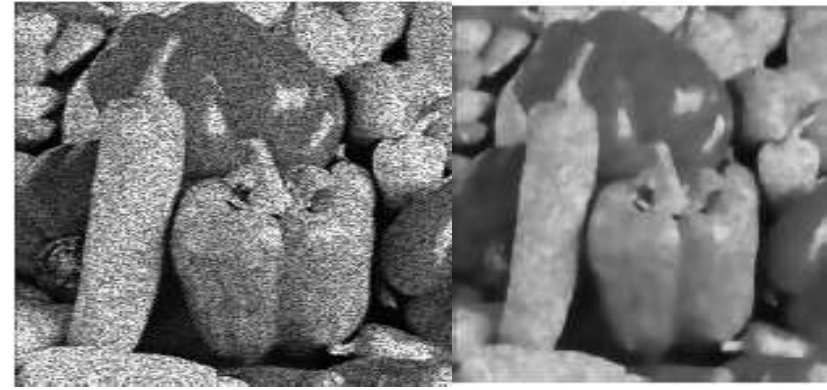

Figure (a) Noisy Signal (b) AA Method

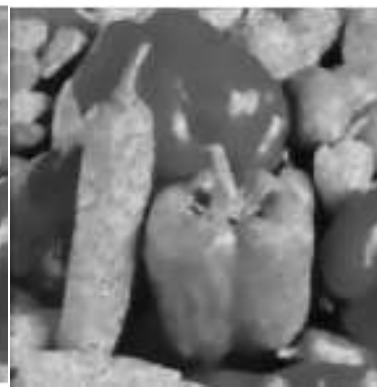

(c) Proposed Method

Fig.7: (a) Noisy Signal, $\sigma^{2}=0.1$. (b) Restored Signal, AA-Method, $\lambda=35$ (c) Restored Signal, Proposed Method, $\alpha_{1}=33, \alpha_{2}=$ $0.00001, \alpha_{3}=600$.

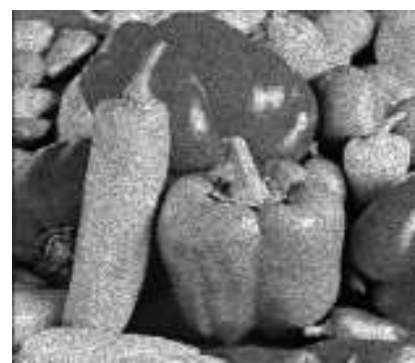

(a) Noisy Image

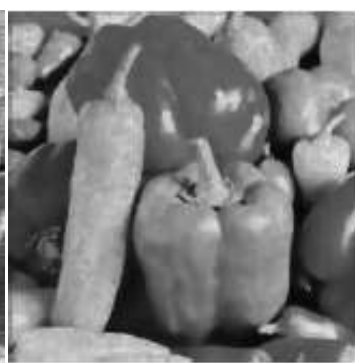

(b) AA-Method

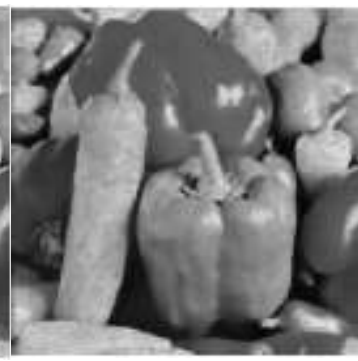

(c) Proposed Method

Fig.8: (a) Noisy Signal, $\sigma^{2}=0.03$. (b) Restored Signal, AA-Mode, $\lambda=35$ (c) Proposed Method, $\alpha_{1}=200, \alpha_{2}=0.0002, \alpha_{3}=$ 600.

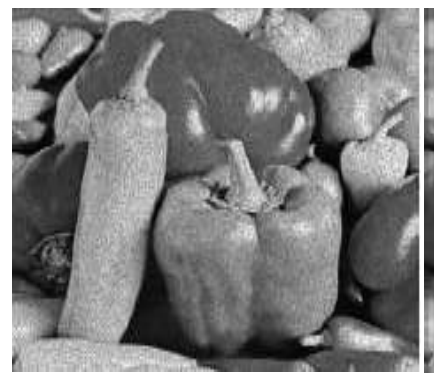

(a) Noisy Image

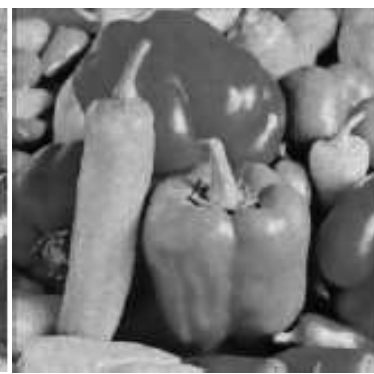

(b) AA-Method

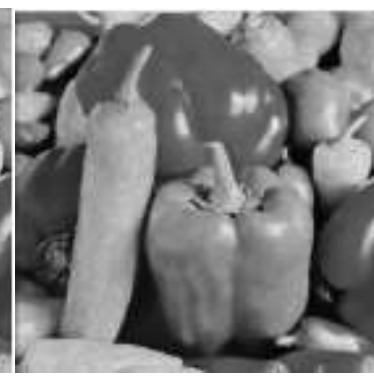

(c) Proposed Method

Fig.9: (a) Noisy Signal, $\sigma^{2}=0.01$. (b) Restored Signal, AA-Method, $\lambda=35$ (c) Restored Signal, Proposed Method with $\alpha_{1}=$ $200, \alpha_{2}=0.00001, \alpha_{3}=600$. 
Test 3 .

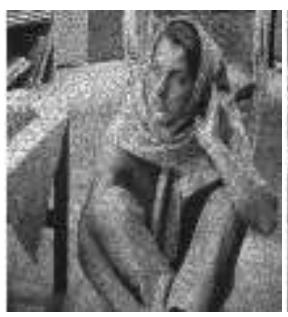

(a)Barbara, 0.03

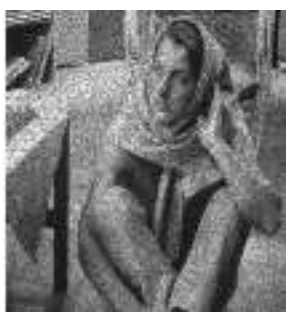

(b) AA Method

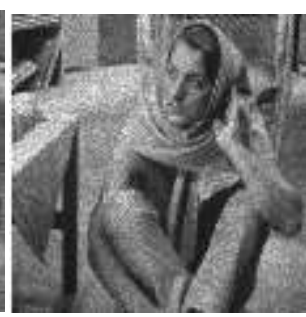

(c) Proposed Method

Fig.10: (a) Noisy Signal, $\sigma^{2}=0.03$ (b) Restored Signal, AA-Method, $\lambda=35$ (c) Restored Signal, Proposed Method with $\alpha_{1}=$ $450, \alpha_{2}=0.0001, \alpha_{3}=650$.

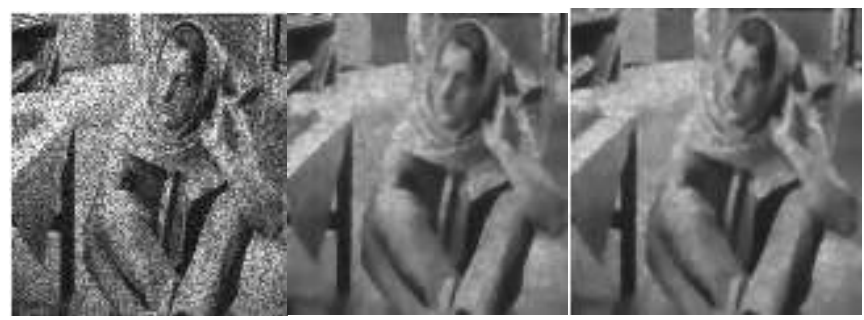
(a) Barbara, 0.1
(b) AA Method
(c) Proposed Method

Fig.11: (a) Noisy Signal, $\sigma^{2}=0.1$ (b) Restored Signal, AA Method, $\lambda=33$ (c) Restored Signal, Proposed Method with $\alpha_{1}=$ $450, \alpha_{2}=0.00001, \alpha_{3}=650$.

Table 1: Restoration results for face images

\begin{tabular}{|l|l|l|l|l|}
\hline \multirow{4}{*}{ Image } & $\begin{array}{c}\text { Variance } \\
" \sigma^{2 "}\end{array}$ & $\begin{array}{c}\text { Fractional Order } \\
\alpha\end{array}$ & $\begin{array}{c}\text { AA-Model } \\
\text { PSNR }\end{array}$ & $\begin{array}{c}\text { Proposed Model } \\
\text { PSNR }\end{array}$ \\
\hline \multirow{3}{*}{$\begin{array}{l}\text { Face } \\
\text { Size }(256 \times 256)\end{array}$} & 0.3 & 1.9 & 26.035 & 27.193 \\
\cline { 2 - 5 } & 0.1 & 1.1 & 28.525 & 29.009 \\
\cline { 2 - 5 } & 0.03 & 1.9 & 31.934 & 33.340 \\
\cline { 2 - 5 } & 0.01 & 1.9 & 35.037 & 36.420 \\
\hline
\end{tabular}

Table 2: Restoration results for Peppers images

\begin{tabular}{|l|l|c|c|c|}
\hline \multirow{3}{*}{ Image } & $\begin{array}{c}\text { Variance } \\
" \sigma^{2 n}\end{array}$ & $\begin{array}{c}\text { Fractional Order } \\
\alpha\end{array}$ & $\begin{array}{c}\text { AA-Model } \\
\text { PSNR }\end{array}$ & $\begin{array}{c}\text { Proposed Model } \\
\text { PSNR }\end{array}$ \\
\hline \multirow{3}{*}{$\begin{array}{c}\text { Peppers } \\
\text { Size }(256 \times 256)\end{array}$} & 0.1 & 1.1 & 26.152 & 26.301 \\
\cline { 2 - 5 } & 0.03 & 1.1 & 29.228 & 29.355 \\
\cline { 2 - 5 } & 0.01 & 1.1 & 32.142 & 32.201 \\
\hline
\end{tabular}

Table 3: Restoration Results for Barbara Images.

\begin{tabular}{|l|c|c|c|c|}
\hline & Variance & Fractional Order & AA-Model & Proposed Model \\
Image & $\sigma^{2 "}$ & $\alpha$ & PSNR & PSNR \\
\hline Barbara & 0.03 & 1.1 & 28.162 & 28.211 \\
\cline { 2 - 5 } Size $(256 \times 256)$ & 0.1 & 1.1 & 25.821 & 25.910 \\
\hline
\end{tabular}

In first experiment we test two dimensional signal "face" $256 \times 256$. We have compared the denoising capabilities of the proposed algorithm with fractional order AA method. Numerous values of $\lambda$ have been tested for fractional order AA algorithm and the best amongst them are mentioned in this paper. As the regularization parameters perform well in denoising so we have chosen the three regularization parameters for increasing the PSNR. In Table 1 we have mentioned the highest PSNR of fractional order AA method and the proposed method. Peppers image of size $256 \times 256$ is 
used to make the second experiment. Best results are chosen among the obtained results of the performed experiements through fractional order AA method. As the quality of the restored image depends upon the regularization parameters so we have chosen the suitable values for regularization parameters. In table 2 we have shown the comparison of our results with those obtained from fractional order AA Algorithm. The PSNR values of the recovered peppers images are listed in table 2 . The values mentioned in table 2 clearly shows that the PSNR values of the proposed method are bigger than fractional order AA-Method. In third test we consider the barbara image. The PSNR values shown in table 3 showing the better performance of the proposed algorithm.

\section{CONCLUSION}

This paper describes a new mathematical approach for image denoising with fractional-order TV. Coupling the Newton algorithmwith time- marching scheme for the solutions of PDEs related to the minmization of denoising model based on fractional order total variation, gives good results.The proposed method can suppress noise very well while it can preserve details of the recovered image. Results obtained from experiements show that the efficiency of the proposed approach are better than what obtained from other existed total variation restoration methods.

\section{REFERENCES}

[1] Rudin L, Osher S, and Fatemi E, "Nonlinear Total Variation Based Model Noise Removal Algorithm,'J. Phys, 60:259-268(1992).

[2] A. Chambolle and P.-L. Lions, "Image Recovery via total Variation Minimization and Related Problems," Numerische Mathematik, 76(2):167-188(1997).

[3] L. Rudin, P.-L. Lions, and S. Osher, "Multiplicative Denoising and Deblurring: Theory and Algorithms," in Geometric Level Sets in Imaging, Vision and Graphics, S. Osher and N. Paragios, Eds., Springer, New York, NY, USA, 103-119(2003).

[4] G. Aubert, J.-F. Aujol, "A Variational Approach to Removing Multiplicative Noise," SIAM Journal on Applied Mathematics, 68(4):925-946(2008).

[5] You Y. L, Kaveh M.,"Fourth-Order Partial Differential Equations for Noise Removal,"IEEE Transactions on Image Processing, 9(10): 1723-1730(2000).

[6] Didas S., Weicket J. and burgeth B,"Properties of Higher Order Nonlinear Diffusion Filtering," Journal of Mathematical Imaging and Vision, 35(3):208-226(2009).

[7] Lysaker M., Lundrevold A., Tai X.C., "Noise Removal Using Fourth Order Partial Differential Equation with
Applications to Medical Magnetic Resonance Images in Space and Time," IEEE Transactions on Image Processing, 12(12): 1579-1590(2003).

[8] Bai J., Feng X. C.,"Fractional-Order Anisotropic Diffusion for Image Denoising,'IEEE Transactions on Image Processing, 16(10): 2492-2502(2007).

[9] Zhang J. and Wei Z. H., "A Class of Fractional-Order Multi-scale Variational Models and Alternating Projection Algorithm for Image Denoising," Applied Mathematical Modeling, 35(5): 2516-2528(2011).

[10] Chambolle A., "An algorithm for Total Variation Minimization and Applications,"Journal of Mathematical Imaging and Vision, 20(1): 89-97(2004).

[11] Y.-M. Haung, M. K. Ng, and Y.-W. Wen, “A New Total Variation Method for Multiplicative Noise Removal", SIAM Journal on Imaging Sciences, 2(1):20-40(2009). 